\title{
Oral Treatment with Probiotics Reduces Allergic Symptoms in Ovalbumin-Sensitized Mice: A Bacterial Strain Comparative Study
}

\author{
S. Hougee ${ }^{a}$ A.J.M. Vriesema ${ }^{a}$ S.C. Wijering ${ }^{c}$ L.M.J. Knippels ${ }^{a} \quad$ G. Folkerts $^{b}$ \\ F.P. Nijkamp ${ }^{\mathrm{b}} \quad$ J. Knol ${ }^{\mathrm{a}} \quad$ J. Garssen ${ }^{\mathrm{a}, \mathrm{b}}$ \\ a Danone Research - Centre for Specialised Nutrition, Wageningen, ${ }^{b}$ Department of Pharmacology \\ and Pathophysiology, Utrecht Institute for Pharmaceutical Sciences, Utrecht University, Utrecht, and \\ 'Numico Research, Wageningen, The Netherlands
}

\section{Key Words}

Ovalbumin-allergic mouse model · Allergic inflammation •

Bifidobacterium breve M-16V · Probiotics

\begin{abstract}
Background/Aim: Evidence demonstrating an important role of the intestinal microbiota in the incidence of allergic disorders has led to the concept of using probiotics as possible antiallergic therapy. This study aimed to select a bacterial strain with the best antiallergic treatment effects from a panel of 6 bacterial strains in a mouse model of ovalbumin(OVA)-allergic asthma. Methods: OVA-sensitized $B A L B / C$ mice were orally administered the bacterial strains Bifidobacterium breve M-16V, B. infantis NumRes251, B. animalis NumRes252 and NumRes253, Lactobacillus plantarum NumRes8 and L. rhamnosus NumRes6. After challenge by OVA inhalation in the lungs, the response to methacholine was measured. Pulmonary inflammation was assessed by analyzing bronchoalveolar lavage fluid for the presence of eosinophils, neutrophils, macrophages and lymphocytes and for interleukin 4, interleukin 5, interleukin 10 and interferon- $\gamma$. OVA-specific lgE, lgG1 and lgG2a were measured in serum. Next, the effect on acute allergic skin reaction was measured after treatment with $B$. breve $\mathrm{M}-16 \mathrm{~V}$ and $L$. plantarum NumRes8. Results: Of the panel of 6 strains, B. breve M$16 \mathrm{~V}$ and L. plantarum NumRes8 inhibited (1) the response to
\end{abstract}

methacholine, (2) reduced the number of eosinophils in the bronchoalveolar lavage fluid, (3) reduced both OVA-specific $\lg E$ and (4) OVA-specific IgG1, whereas the other strains did not affect all these parameters simultaneously. B. breve $M$ $16 \mathrm{~V}$ but not $L$. plantarum NumRes 8 reduced interleukin 4, interleukin 5 and interleukin 10. Furthermore, B. breve M-16V but not $L$. plantarum NumRes8 reduced acute allergic skin reactions to OVA. Conclusion: $B$. breve M-16V was identified as the most potent antiallergic strain.

Copyright $\odot 2009$ S. Karger AG, Basel

\section{Introduction}

The incidence of allergic disorders has dramatically increased over the past decades such that these disorders are the most common chronic diseases of childhood in the developed world nowadays $[1,2]$. This rise in the incidence of allergies has been linked with epidemiological evidence of a reduced microbial burden, the 'hygiene hypothesis' and new insights thereof [3-6]. Interestingly, the composition of the intestinal microbiota has also been associated with the incidence of allergies. The intes-

Danone Research - Centre for Specialised Nutrition is formerly known as Numico Research.

\section{KARGER}

Fax +41613061234 E-Mail karger@karger.ch www.karger.com
(C) 2009 S. Karger AG, Basel $1018-2438 / 10 / 1512-0107 \$ 26.00 / 0$

Accessible online at:

www.karger.com/iaa
Correspondence to: Dr. Sander Hougee

Danone Research - Centre for Specialised Nutrition

PO Box 7005

NL-6700 CA Wageningen (The Netherlands)

Tel. +31 317467 800, Fax +31 317466 500, E-Mail sander.hougee@danone.com 
tine of allergic infants was less often colonized by lactobacilli and bifidobacteria, whereas it was more often colonized by aerobic microorganisms $[7,8]$. Infants with atopic dermatitis (AD) had reduced levels of fecal bifidobacteria as well [9]. In addition, the reduced levels of bifidobacteria preceded the development of atopic disease in Finnish infants [10]. Furthermore, it is generally recognized that the gut microbiota contribute to normal immune maturation in early life $[11,12]$. These findings have all contributed to the concept of modulating the intestinal microbiota for antiallergic therapy by adding prebiotics, probiotics, or a combination (synbiotics) to the diet.

Probiotics are defined as live microorganisms which, when administered in adequate amounts, confer a health benefit on the host [13]. Evidence is accumulating that specific probiotics may counterbalance the predominance of the proallergic, T-helper (Th) 2-directed immune response [14-19]. Currently, there is more convincing evidence for a preventive effect than a therapeutic effect of probiotics according to a recent meta-analysis of clinical trials of probiotics for the prevention and treatment of pediatric AD [20]. In this meta-analysis, clinical studies were summarized that used several bacterial strains or combinations of bacterial strains. This is in contradiction with a more recent review article in which it is concluded that probiotics cannot be generally recommended for the primary prevention of atopic diseases [21]. Some of the confusion on whether probiotics are effective is illustrated with the following clinical trials in which infants were treated with Lactobacillus rhamnosus GG (LGG) for AD: The first report showed encouraging results, i.e., the frequency of $\mathrm{AD}$ was reduced by half in infants that were given LGG. It is noteworthy that this study was conducted in infants with a family history of atopic disease, and that mothers of these infants started supplementation with LGG 2-4 weeks before the expected delivery [22]. A clinical trial in which infants with suspected cow's milk allergy were treated with LGG did not show any effect on $\mathrm{AD}$ in the whole group; $\mathrm{AD}$ was reduced in the IgE-sensitized infants, but not in the nonIgE-sensitized infants [23]. A recent trial studied the effect of LGG supplementation in infants suffering from mild to moderate AD and showed no treatment effect of LGG in this population [24]. Possible explanations include differences between preventive and therapeutic effects of probiotics and sensitization to IgE as factors that determine the antiallergic effects of LGG. However, a more recent trial that aimed to repeat the first report on reducing $\mathrm{AD}$ with $\mathrm{LGG}$ was not successful: no reduction of AD with LGG was observed in exactly the same setup as in the first report [25], suggesting that other factors play a role. Preclinically, a preventive effect of LGG in reducing allergy is supported by studies in mouse models of allergic airway inflammation: perinatal and prenatal supplementation reduced allergic airway and peribronchial inflammation although airway reactivity to methacholine was not changed [26]. This was in contrast with a study in mice that received LGG from birth every other day and were sensitized to ovalbumin (OVA) on days 29, $31,33,36,38$ and 40 ; on day 56 , all aspects of the asthmatic phenotype were suppressed, including airway reactivity to methacholine in comparison with mice that did not receive LGG [27].

Next to the difference in outcome with the same bacterial strain in different supplementation protocols in preclinical and clinical trials, it is generally recognized that the biological effects can depend on the specific bacterial strain that is used. Consequently, specific health effects should be demonstrated for each bacterial strain. Kopp and Salfeld [21] conclude that probiotics cannot be generally recommended for primary prevention, but that the concept of using probiotics for primary prevention of allergy still seems plausible. However, the selection of the most beneficial probiotic strain or the composition of different probiotics and/or prebiotics, the timing of supplementation, the optimal dose and method of administration still need to be determined. The present study is performed in search for the most beneficial probiotic strain of a panel of 6 for its antiallergic treatment effects in a mouse model of OVA-allergic asthma. Four strains of bifidobacteria and two strains of lactobacilli are compared. The strain with the best antiallergic effects will be a candidate for a clinical study with already allergic sensitized humans in whom the inhibition of allergic symptoms will be studied.

\section{Materials and Methods}

\section{Animals}

Male BALB/cByJIco-specified, pathogen-free mice (5-8 weeks; 20-25 g; Charles River, Someren, The Netherlands) were accustomed to their new environment for at least 1 week before the start of the experiment. Animals were housed in type III macrolon cages and provided ad libitum with standard rodent feed (Hopefarms, Woerden, The Netherlands) and tap water. Temperature and humidity were kept constant at $22^{\circ} \mathrm{C}$ and $50 \%$, respectively. All experiments were approved by an independent animal experiments committee according to Dutch laws on animal experimentation. 


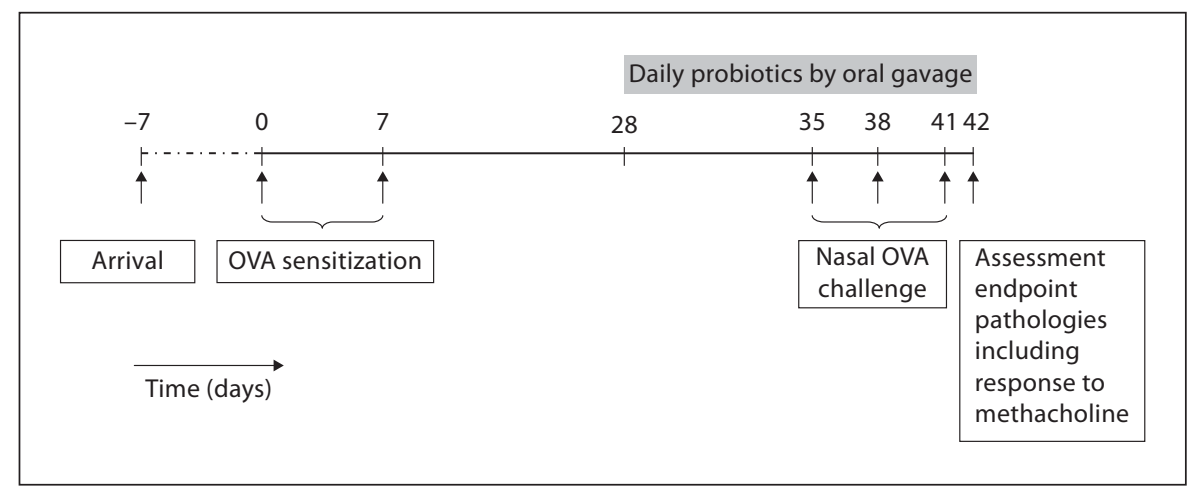

Fig. 1. Time line of the mouse OVA allergy experiment. Mice were acclimatized to their new accommodation 1 week before the first sensitization on day 0 . Sensitizations were performed on days 0 and 7 by injecting the mice intraperitoneally with $10 \mu \mathrm{g}$ OVA in alum. Daily administration of $10^{9} \mathrm{CFUs} \mathrm{probiotics} \mathrm{by} \mathrm{oral} \mathrm{gavage}$ started on day 28 (treatment protocol) and continued until day 42.
On days 35, 38 and 41, the animals were nasally challenged with $10 \mathrm{mg} / \mathrm{ml}$ aerosolized OVA. The airway response to methacholine was measured at day 42 , from which mice were exposed to increasing dosages of methacholine. Subsequently, the mice were sacrificed with a lethal dose of anesthetics, after which blood and BALF were collected.

\section{OVA Sensitization}

Mice were sensitized to OVA by 2 intraperitoneal injections with $10 \mu \mathrm{g}$ OVA grade V (Sigma Aldrich, Zwijndrecht, The Netherlands) in $2.25 \mathrm{mg} / 0.1 \mathrm{ml}$ AlumImject (Pierce, Perbio Science Nederland, Etten-Leur, The Netherlands) in phosphate-buffered saline (PBS) on days 0 and 7. Sham-sensitized animals received $0.1 \mathrm{ml}$ PBS only (fig. 1).

\section{Probiotic Bacteria}

The following probiotic strains, derived from the Numico Research Culture Collection, were selected for testing: Bifidobacterium animalis subsp. lactis NumRes252, B. animalis subsp. lactis NumRes253, B. breve M-16V (Morinaga Milk Industry Co., Ltd., Zama, Japan), B. infantis NumRes251, Lactobacillus plantarum NumRes8 and L. rhamnosus NumRes6. The bacteria were cultured in MRS broth (Oxoid, Basingstoke, UK) and resuspended in PBS to the right dose prior to use. The species level of the strains was confirmed by double-stranded $16 \mathrm{~S}$ rDNA sequencing [28].

Internally transcribed spacer polymerase chain reaction (ITSPCR) was used to genetically discriminate between the two $B$. animalis subsp. lactis strains, followed by restriction analysis with HindIII or PstI (Roche Diagnostics BV, Almere, The Netherlands) [29].

\section{Probiotic Treatment}

From day 28 onwards, $10^{9}$ colony forming units (CFUs) of the probiotic strains were administered per animal per day. Probiotic strains were suspended in $0.4 \mathrm{ml}$ of PBS and given by oral gavage up to the end of the experiment (day 42). Control animals received solely PBS as placebo. Groups consisted of 7-18 animals (fig. 1).

\section{OVA Aerosol Challenge}

OVA-sensitized mice were exposed to $10 \mathrm{mg} / \mathrm{ml}$ nebulized OVA aerosols in PBS using Pari LC Star (Pari IS-2, Pari-Werk $\mathrm{GmbH}$, Starnberg, Germany) in an aerosol cabin for $20 \mathrm{~min} 3$ times on days 35,38 and 41 (fig. 1).

\section{Airway Response to Methacholine}

The response to methacholine was analyzed $24 \mathrm{~h}$ after the last OVA aerosol challenge on day 42 of the experiment. For the analysis of the response to methacholine, conscious unrestrained mice were exposed to increasing dosages of aerosolized methacholine $(1.56-50 \mathrm{mg} / \mathrm{ml})$. The response to methacholine was investigated using whole-body plethysmography (Buxco, Emka technologies, Paris, France). The response to methacholine is expressed as enhanced pause $\left(\mathrm{P}_{\mathrm{enh}}\right)$, as described in detail previously [30]. After the last measurement, the animals were sacrificed with a lethal intraperitoneal injection of sodium pentobarbital (300 mg/kg body weight).

\section{Bronchoalveolar Lavage}

Lungs were rinsed with $1 \mathrm{ml}$ of pyrogen-free saline $(0.9 \%$ $\mathrm{NaCl}$ ) supplemented with 1 protease inhibitor cocktail tablet per $100 \mathrm{ml}$ (Roche Diagnostics, Mannheim, Germany) and $4 \mathrm{~g} / 100 \mathrm{ml}$ of bovine serum albumin (BSA) at $37^{\circ} \mathrm{C}$ in situ. The rinsing procedure was repeated 3 times with $1 \mathrm{ml}$ of $0.9 \% \mathrm{NaCl}$ without protease inhibitors. Supernatants of the first collected milliliter were stored at $-30^{\circ} \mathrm{C}$ for cytokine analysis. Cell preparations of the bronchoalveolar lavage fluid (BALF) were made by cytospinning the cells onto glass for $5 \mathrm{~min}$ at $400 \mathrm{~g}$ at $4^{\circ} \mathrm{C}$. Cytospin cell preparations were stained by DiffQuick (Merz \& Dade AG, Düdingen, Switzerland). Numbers of eosinophils, macrophages, neutrophils and lymphocytes were scored by light microscopy in a blinded setup.

\section{Determination of Serum Levels of Immunoglobulins and BALF Cytokines}

Blood was collected by cardiac puncture immediately after the lethal injection of anesthetics. The blood was coagulated for $1 \mathrm{~h}$ at room temperature and subsequently centrifuged for $5 \mathrm{~min}$ at $17,500 \mathrm{~g}$. Serum was stored at $-80^{\circ} \mathrm{C}$ until further analysis. The analyses of OVA-specific immunoglobulin (Ig) E, IgG1 and IgG2a (Pharmingen, San Diego, Calif., USA) were done as described 


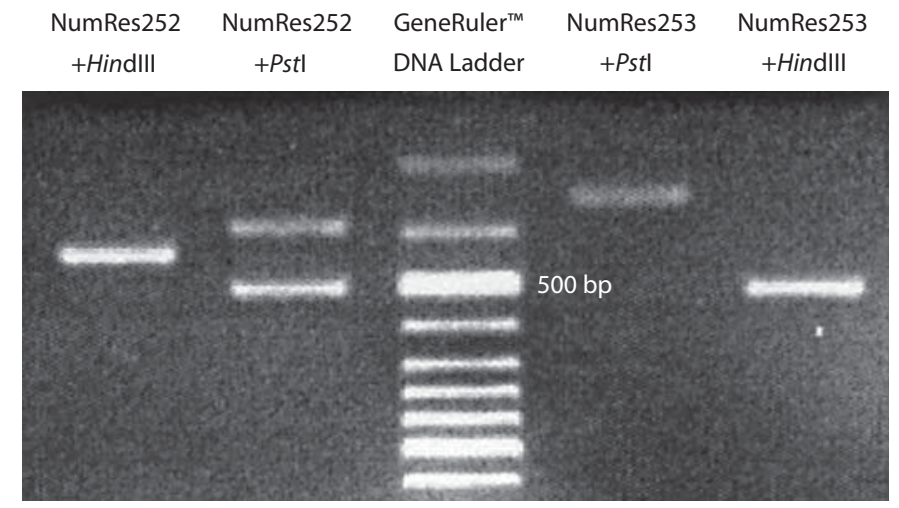

Fig. 2. Discrimination between the 2 B. animalis subsp. lactis strains NumRes252 and NumRes253 after restriction digestion of the amplified ITS regions using PstI or HindIII and separation on a $2 \%$ agarose gel and ethidium bromide staining.

elsewhere [31], using rat antimouse IgE, IgG1 and IgG2a capture monoclonal antibodies, respectively (Pharmingen). The cytokines interleukin (IL) 4, IL-5, IL-10, interferon (IFN)- $\gamma$ were determined by ELISA (BioSource International, Inc., Camarillo, Calif., USA) according to the supplier's protocol.

\section{OVA-Specific Skin Response}

The above-mentioned experiments were performed at the research facilities of Utrecht Institute for Pharmaceutical Sciences. At another laboratory (Centre for Small Laboratory Animals, Wageningen University, Wageningen, The Netherlands), the same experimental setup was followed, except for the response to methacholine, which was replaced by the OVA-specific skin response. Only the 2 bacterial strains that demonstrated both reduction in the response to methacholine and in eosinophil influx in the BALF were evaluated (B. breve M-16V and L. plantarum NumRes8). The acute OVA-specific skin response was measured after intradermal injection of OVA into the pinnae of both ears. Basal ear thickness was measured and, subsequently, $20 \mu \mathrm{l} \mathrm{OVA}$ in PBS $(0.1 \mathrm{mg} / \mathrm{ml})$ was injected intradermally. One hour after OVA injection into the ears, ear thickness was measured again. Ear thickness was measured in duplicate per ear using a digital micrometer (Mitutoyo, Veenendaal, The Netherlands). Basal ear thickness was subtracted from ear thickness $1 \mathrm{~h}$ after OVA injection into each mouse, and the average ear swelling of the different treatment groups was expressed. The mice were anesthetized with isoflurane $/ \mathrm{O}_{2}$ for all ear thickness measurements.

\section{Analyses and Statistical Calculations}

Data are expressed as arithmetic means \pm standard error of the mean (SEM). An unpaired Student's $t$ test was performed for statistical comparisons between 2 groups, unless stated otherwise. The airway response curves to methacholine were analyzed by a general linear model of repeated measurements, followed by post hoc comparison between groups (Dunnett's test). Data were $\log 10$-transformed before analysis to equalize variances in all groups. A probability value of $\mathrm{p}<0.05$ was considered to be significant.

\section{Results}

Consistently Reduced Response to Methacholine with Oral Treatment of B. breve $M-16 \mathrm{~V}$

The response to methacholine was enhanced in the OVA-allergic group that received PBS as a control oral treatment compared to the non-OVA-allergic sham-sensitized control group that received oral PBS treatment. This difference in the response to methacholine between the allergic and the non-allergic mice is significant at the lowest dose of $0 \mu \mathrm{g} / \mathrm{ml}$ methacholine and persisted up to $50 \mu \mathrm{g} / \mathrm{ml}$ (fig. 3).

In contrast to the other tested bifidobacteria, only the oral treatment with $B$. breve M-16V significantly reduced the increased airway response to methacholine at the highest concentration of methacholine. Moreover, the response to methacholine after oral treatment with B. breve $\mathrm{M}-16 \mathrm{~V}$ was consistently reduced at all methacholine concentrations and reached statistical significance at $25 \mu \mathrm{g} /$ $\mathrm{ml}$ methacholine.

Oral treatment with L. rhamnosus NumRes6 as well as with $L$. plantarum NumRes8 significantly reduced the increased response to methacholine at the 2 highest concentrations of methacholine, i.e., 25 and $50 \mu \mathrm{g} / \mathrm{ml}$. At the lower concentrations of methacholine, the airway response was similar to that of the PBS-treated group.

Comparing the bifidobacteria with the lactobacilli, $B$. breve M-16V displayed the best overall reduction in the increased response to methacholine at all concentrations.

\section{Reduced Eosinophil and Lymphocyte Influx into the}

Airways with B. breve M-16V

Eosinophil, macrophage, neutrophil and lymphocyte cell counts in the BALF were all elevated in the OVAallergic PBS-treated group compared to the non-OVAallergic PBS-treated sham-sensitized group. The number of eosinophils in the BALF was significantly reduced after treatment with either $B$. breve $\mathrm{M}-16 \mathrm{~V}$ or $L$. plantarum NumRes8, but not with $B$. animalis NumRes252, B. animalis NumRes253, B. infantis NumRes251 or with L. rhamnosus NumRes6, compared to the PBS-treated OVA-allergic group. In addition, the number of lymphocytes was reduced after treatment with $B$. breve M-16V compared to PBS treatment in OVA-allergic mice. Treatment with $L$. plantarum NumRes8 did not reduce lymphocyte numbers in BALF, nor did treatment with the other bacteria. The number of macrophages and neutrophils was not affected by any of the treatments (fig. 4). 
Fig. 3. The airway response to aerosolized methacholine measured $24 \mathrm{~h}$ after the last OVA challenge in mice as expressed by $\mathrm{P}_{\text {enh }}$. The results are expressed as arithmetic means ( $n=7-18$ per group); the table shows the mean \pm SEM airway responses for all methacholine concentrations. ${ }^{\text {a Sta- }}$ tistically significant difference $(p<0.05)$ compared to sham-sensitized animals; b statistically significant difference $(\mathrm{p}<$ $0.05)$ compared to the PBS-treated, sensitized mice. a Bifidobacteria: B. animalis subsp. lactis Num Res253, B. breve M-16V, B. animalis subsp. lactis NumRes 252 and B. infantis NumRes251. b Lactobacilli: $L$. rhamnosus NumRes6 and L. plantarum NumRes8.

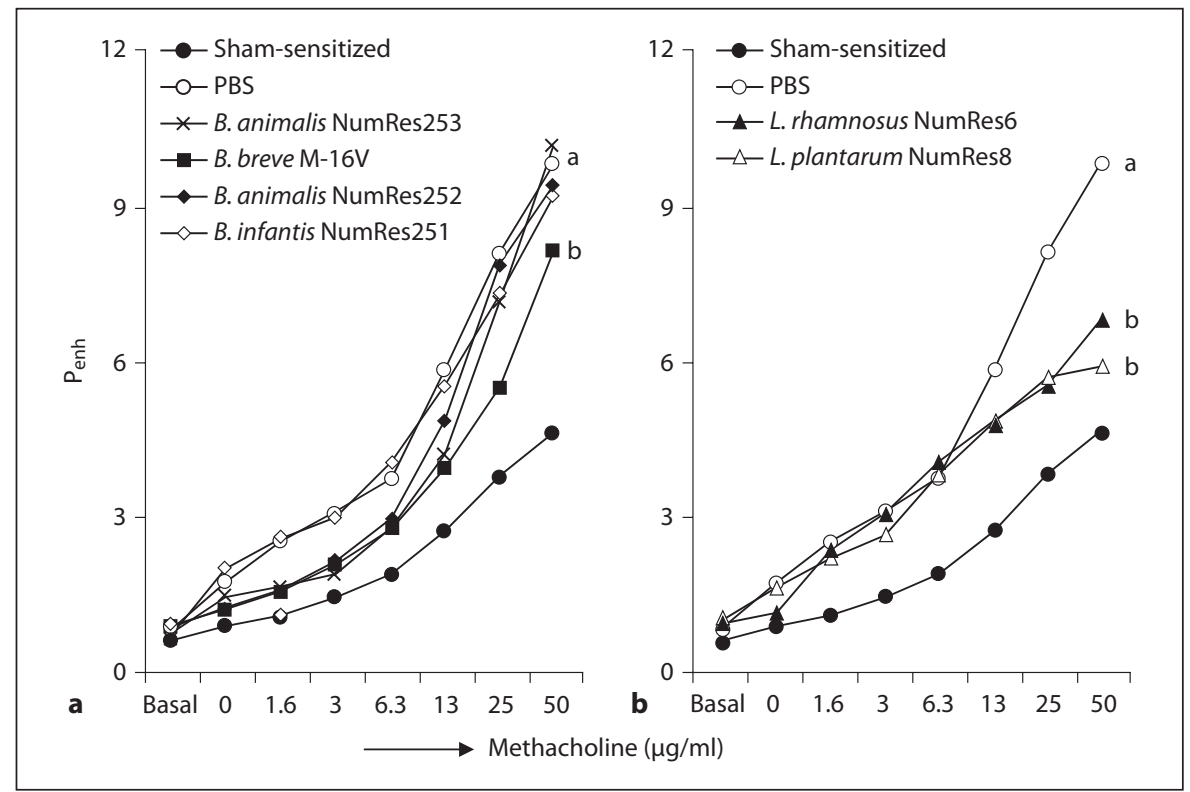

\begin{tabular}{|c|c|c|c|c|c|c|c|c|}
\hline & \multicolumn{8}{|c|}{ Methacholine } \\
\hline & \multicolumn{2}{|c|}{$0 \mu \mathrm{g} / \mathrm{ml}$} & \multicolumn{2}{|c|}{$6.3 \mu \mathrm{g} / \mathrm{ml}$} & \multicolumn{2}{|c|}{$25 \mu \mathrm{g} / \mathrm{ml}$} & \multicolumn{2}{|c|}{$50 \mu \mathrm{g} / \mathrm{ml}$} \\
\hline & mean & SEM & mean & SEM & mean & SEM & mean & SEM \\
\hline Sham-sensitized & 0.9 & 0.1 & 1.9 & 0.2 & 3.8 & 0.5 & 4.6 & 0.5 \\
\hline PBS & $1.7^{\mathrm{a}}$ & 0.2 & $3.7^{\mathrm{a}}$ & 0.4 & $8.1^{\mathrm{a}}$ & 0.7 & $9.8^{\mathrm{a}}$ & 0.8 \\
\hline B. animalis NumRes 253 & 1.4 & 0.2 & 2.8 & 0.4 & 7.2 & 1.0 & 10.1 & 1.3 \\
\hline B. breve $\mathrm{M}-16 \mathrm{~V}$ & 1.2 & 0.1 & 2.8 & 0.3 & $5.5^{\mathrm{b}}$ & 0.7 & $8.1^{\mathrm{b}}$ & 0.9 \\
\hline B. animalis NumRes 252 & 1.2 & 0.1 & 3.0 & 0.5 & 7.9 & 1.3 & 9.4 & 1.2 \\
\hline B. infantis NumRes 251 & 2.0 & 0.3 & 4.1 & 0.7 & 7.3 & 1.4 & 9.2 & 1.5 \\
\hline L. rhamnosus NumRes6 & 1.1 & 0.2 & 4.1 & 0.6 & $5.5^{\mathrm{b}}$ & 0.7 & $6.8^{\mathrm{b}}$ & 0.7 \\
\hline L. plantarum NumRes8 & 1.6 & 0.5 & 3.8 & 0.6 & $5.6^{\mathrm{b}}$ & 0.5 & $5.8^{\mathrm{b}}$ & 0.9 \\
\hline
\end{tabular}

Cytokine Levels in BALF: Reduction in IL-4, IL-5 and IL-10 by B. animalis NumRes 252 or B. breve $M-16 \mathrm{~V}$

Levels of IL-4, IL-5 and IL-10 were significantly elevated in BALF of OVA-allergic PBS-treated mice compared to the non-OVA-allergic PBS-treated mice. The level of IFN- $\gamma$ was enhanced as well, although not statistically significantly. Measured cytokines in BALF were not affected by treatment with lactobacilli in comparison to the OVA-allergic PBS-treated mice. Treatment with $B$. animalis NumRes 252 or B. breve M-16V significantly reduced the level of IL- 4 to around 20 and $60 \%$ of the OVA-allergic PBS-treated mice, respectively. Treatment with $B$. animalis NumRes 253 or B. infantis NumRes251 resulted in a nonsignificant reduction of IL-4 levels in BALF. Treatment with $B$. animalis NumRes253, B animalis NumRes 252 , B. breve M-16V or $B$. infantis NumRes 251 significantly reduced IL-5 levels to around $75-85 \%$ of the OVA-allergic PBS-treated group. Similarly, treatment with B. animalis NumRes253 significantly reduced IL-10 levels in the BALF to about $25 \%$ of OVA-allergic PBS-treated mice. Treatment with B. breve $\mathrm{M}-16 \mathrm{~V}, B$. animalis NumRes 252 or $B$. infantis NumRes251 significantly lowered the level of IL-10 to around $40-50 \%$ of OVA-allergic PBS-treated mice. The level of IFN- $\gamma$ in BALF was not significantly affected by any of the treatments with either bifidobacteria or lactobacilli (fig. 5).

OVA-Specific Antibody Titers in Serum: Reduction in OVA-IgE and OVA-IgG1 with B. animalis NumRes253, B. breve $M-16 \mathrm{~V}$ or L. rhamnosus NumRes 6

OVA-allergic PBS-treated mice had significantly increased serum titers of OVA-specific IgE, IgG1 and IgG2a compared to non-OVA-allergic PBS-treated sham-sensi- 
Fig. 4. Pulmonary inflammation represented by the influx of specific leukocytes in BALF. Mice in the negative control group were sham-sensitized with PBS. Mice in all other groups were sensitized and challenged with OVA. The positive ent test groups received a different bacterial strain per oral gavage. Total numbers of cells are shown in the graph, whereas the table indicates the mean \pm SEM cell numbers per cell type. ${ }^{a}$ Statistically significant difference $(\mathrm{p}<0.05)$ compared to sham-sensitized animals; ${ }^{\mathrm{b}}$ statistically significant difference $(\mathrm{p}<0.05)$ compared to the PBS-treated, sensitized mice. control group received $\mathrm{PBS}$, and the differ-

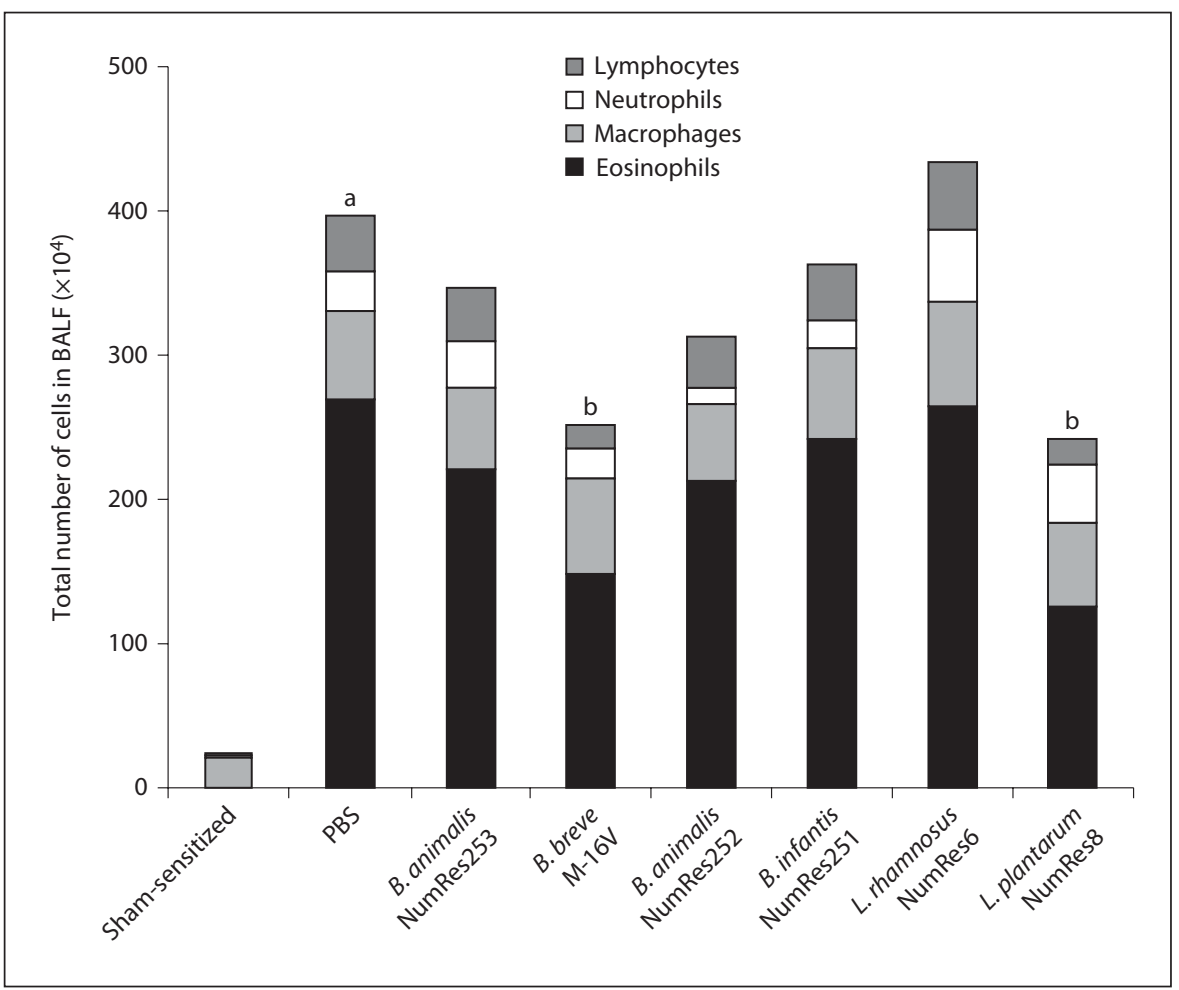

\begin{tabular}{|c|c|c|c|c|c|c|c|c|c|c|}
\hline \multirow[t]{2}{*}{ Treatment } & \multicolumn{2}{|c|}{$\begin{array}{l}\text { Eosinophils } \\
\times 10^{4}\end{array}$} & \multicolumn{2}{|c|}{$\begin{array}{l}\text { Macrophages } \\
\times 10^{4}\end{array}$} & \multicolumn{2}{|c|}{$\begin{array}{l}\text { Neutrophils } \\
\times 10^{4}\end{array}$} & \multicolumn{2}{|c|}{$\begin{array}{l}\text { Lymphocytes } \\
\times 10^{4}\end{array}$} & \multicolumn{2}{|l|}{$\begin{array}{l}\text { Total } \\
\times 10^{4}\end{array}$} \\
\hline & mean & SEM & mean & SEM & mean & SEM & mean & SEM & mean & SEM \\
\hline Sham-sensitized & 0.1 & 0.0 & 22.4 & 2.5 & 0.4 & 0.1 & 1.4 & 0.5 & 24.3 & 1.3 \\
\hline PBS & $268.1^{a}$ & 37.1 & $61.1^{\mathrm{a}}$ & 8.0 & $28.4^{\mathrm{a}}$ & 4.7 & $39.6^{\mathrm{a}}$ & 10.6 & $397.2^{\mathrm{a}}$ & 15.7 \\
\hline B. animalis NumRes 253 & 219.5 & 51.5 & 56.3 & 9.4 & 33.2 & 14.1 & 37.3 & 8.9 & 346.3 & 19.1 \\
\hline B. breve M-16V & $148.3^{b}$ & 23.4 & 65.1 & 10.7 & 23.2 & 6.6 & $15.3^{b}$ & 2.6 & $251.9^{b}$ & 9.5 \\
\hline B. animalis NumRes 252 & 211.9 & 31.2 & 54.0 & 8.2 & 12.2 & 2.7 & 34.4 & 8.8 & 312.5 & 15.8 \\
\hline B. infantis NumRes 251 & 242.9 & 48.2 & 61.8 & 10.1 & 18.8 & 5.4 & 39.1 & 6.9 & 362.6 & 19.7 \\
\hline L. rhamnosus NumRes6 & 263.4 & 48.8 & 73.4 & 15.1 & 50.5 & 15.7 & 45.1 & 12.3 & 432.4 & 21.7 \\
\hline L. plantarum NumRes8 & $127.2^{\mathrm{b}}$ & 41.1 & 57.1 & 15.0 & 40.8 & 10.8 & 17.6 & 7.2 & $242.1^{\mathrm{b}}$ & 12.7 \\
\hline
\end{tabular}

tized mice, indicating that the OVA sensitizations were successful. Oral treatments with the different bifidobacteria or lactobacilli all resulted in significantly reduced OVA-specific IgE titers ranging from 50 to $75 \%$ of the OVA-allergic PBS-treated mice. OVA-specific IgG1 titers were only significantly reduced after oral treatment with either $B$. animalis NumRes $253, B$. breve $\mathrm{M}-16 \mathrm{~V}$ or $L$. rhamnosus NumRes6. OVA-specific IgG2a was not significantly affected by any of the treatments although a trend to higher titers (50-100\% higher) was observed with all treatments (fig. 6).

\section{OVA-Specific Skin Response}

OVA-allergic PBS-treated mice had significantly increased ear swelling when compared to sham-sensitized PBS-treated mice, indicating that the OVA-allergic mice demonstrated an acute allergic skin reaction to OVA. Treatment of OVA-allergic mice with L. plantarum NumRes8 did not result in significantly reduced ear swelling when compared to PBS treatment. Interestingly, treatment with $B$. breve $\mathrm{M}-16 \mathrm{~V}$ significantly reduced the acute allergic skin reaction to OVA compared to the PBStreated OVA-allergic mice (fig. 7). 


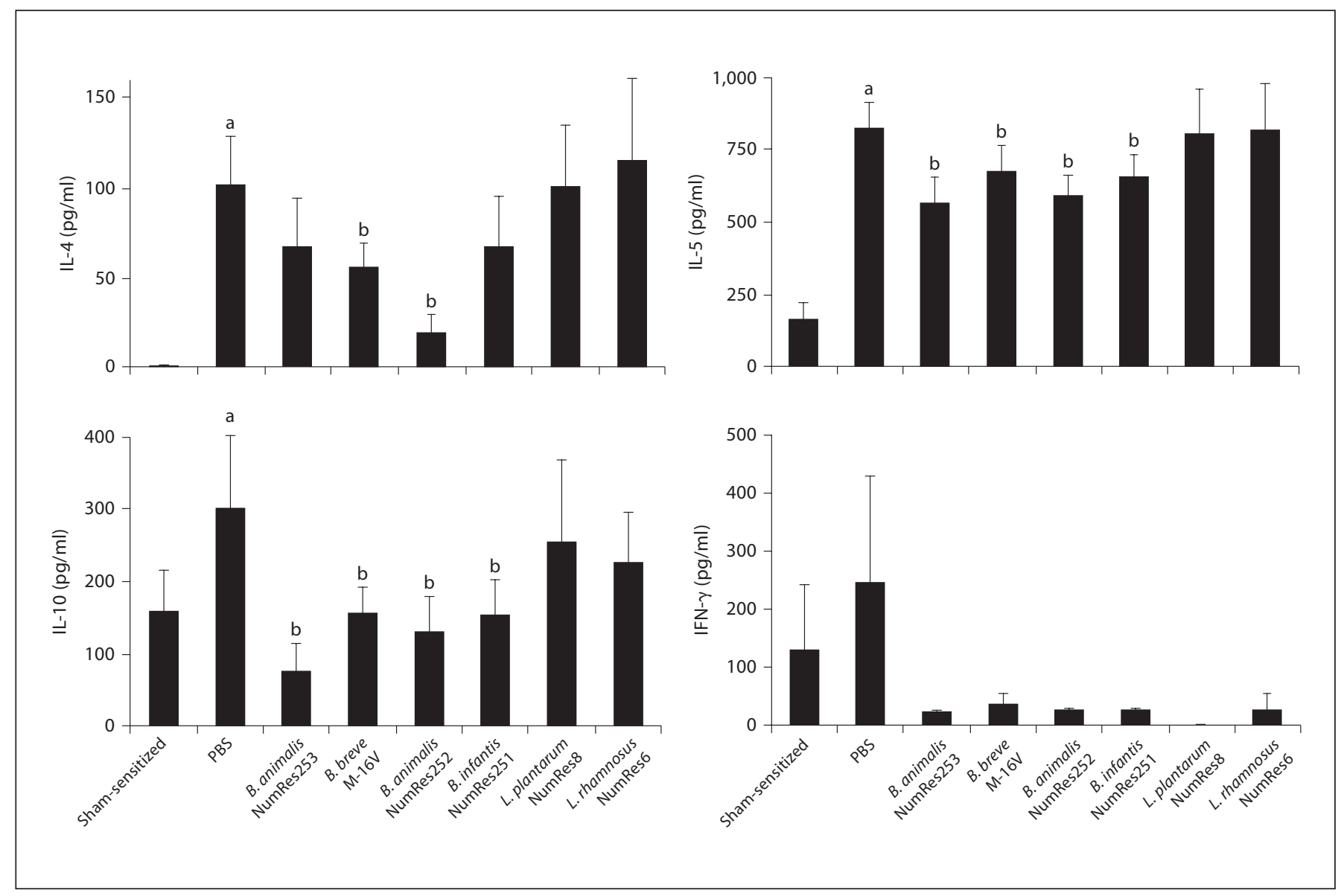

Fig. 5. Pulmonary inflammation represented by the cytokine levels IL-4, IL-5, IL-10 and IFN- $\gamma$ in the BALF. Mice in the negative control group were sham-sensitized with PBS. Mice in all other groups were sensitized and challenged with OVA. The positive control group received PBS and the different test groups received a different bacterial strain per oral gavage. Mean \pm SEM cytokine levels (picograms per milliliter) are shown. ${ }^{\text {a }}$ Statistically significant difference $(\mathrm{p}<0.05)$ compared to sham-sensitized animals; ${ }^{b}$ statistically significant difference $(\mathrm{p}<0.05)$ compared to the PBS-treated, sensitized mice.

\section{Discussion}

In the present study, a well-established mouse model of allergic asthma was used to identify the bacterial strain with the most potent antiallergic effects among a group of 4 bifidobacterium strains (B. animalis NumRes253, $B$. animalis NumRes252, $B$. infantis NumRes251, B. breve $\mathrm{M}-16 \mathrm{~V}$ ) and 2 lactobacillus strains (L. plantarum NumRes8 and L. rhamnosus NumRes6). From the results, it is evident that oral treatment with $B$. breve $\mathrm{M}-16 \mathrm{~V}$ was most effective in reducing the allergic response.

Oral treatment with $B$. breve M-16V most successfully reduced the increased airway response to methacholine when the whole concentration range of methacholine in OVA-sensitized and nasally challenged mice is taken into consideration. Recently, Forsythe et al. [32] demonstrated that oral treatment with L. reuteri of OVA-sensitized mice attenuated airway responsiveness, whereas treatment with $L$. salivarius had no effect on airway responsiveness. However, their experimental setup differed from ours. In their study, daily oral administration of the bacterial strains was initiated on the same day as the second OVA sensitization and was continued for 9 days. This is in contrast to our experimental design, in which the bacterial strains were administered starting 3 weeks after the second OVA sensitization and this was continued for 2 weeks. In the experimental setup of Forsythe et al. [32], it cannot be excluded that the initiation of oral administration with bacterial strains concurrently with the second OVA sensitization modulated the allergic sensitiza- 


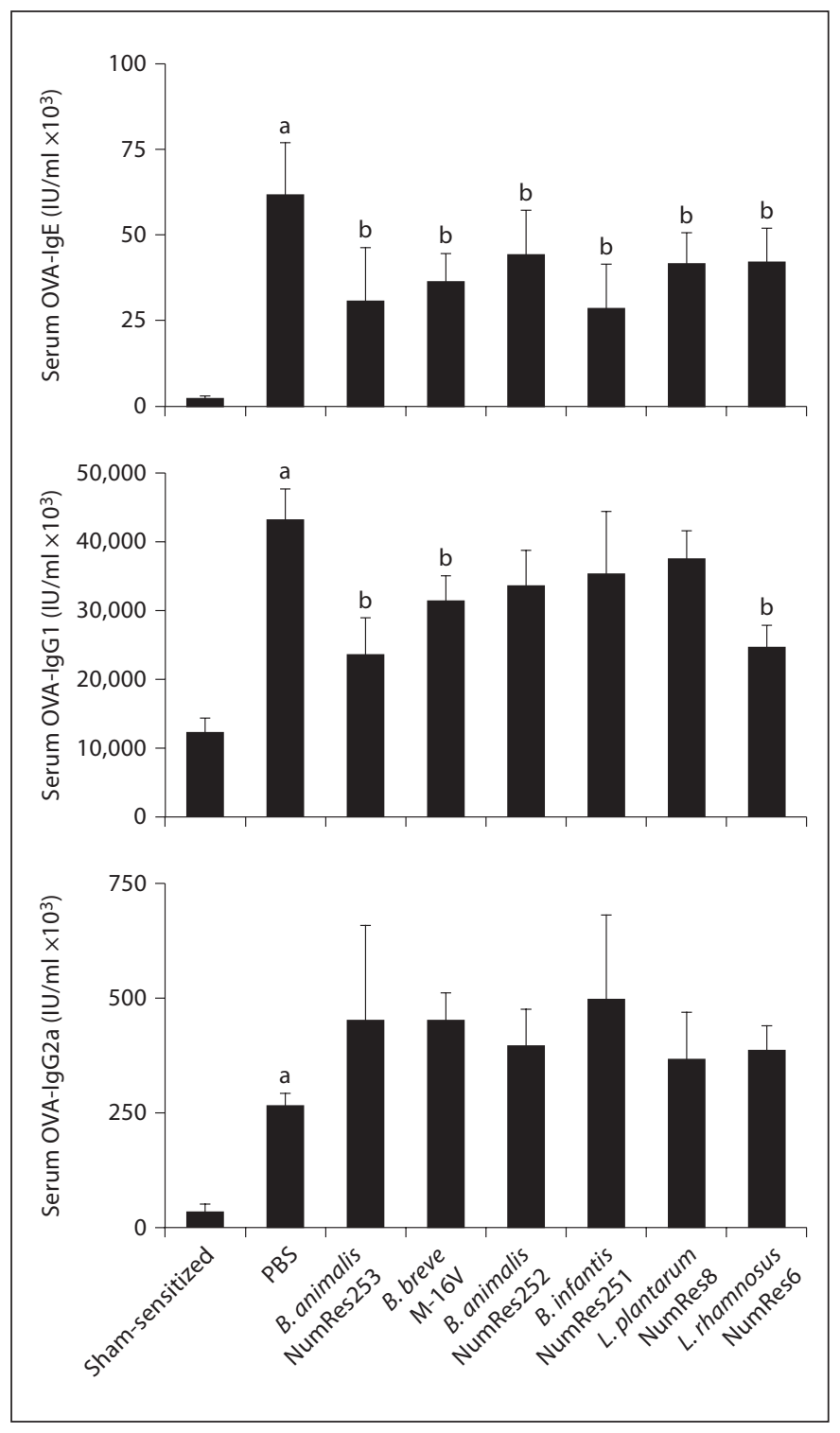

Fig. 6. OVA-specific serum IgE, IgG1 and IgG2a. Mice in the negative control group were sham-sensitized with PBS. Mice in all other groups were sensitized and challenged with OVA. The positive control group received PBS and the different test groups received a different bacterial strain per oral gavage. Mean \pm SEM OVA-Ig levels in international units (IU) per milliliter are shown. a Statistically significant difference $(\mathrm{p}<0.05)$ compared to shamsensitized animals; ${ }^{b}$ statistically significant difference $(\mathrm{p}<0.05)$ compared to the PBS-treated, sensitized mice.

tion to OVA. In the present study, the 3-week interval between the second sensitization and the initiation of oral administration with the bacterial strains should warrant a dedicated 'treatment effect', i.e., a reduction in the increased airway response to methacholine with $B$.

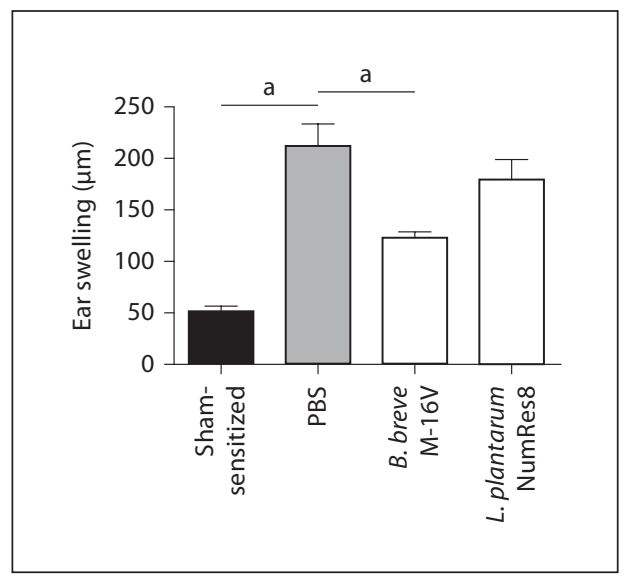

Fig. 7. Acute allergic skin reactions expressed by ear swelling $1 \mathrm{~h}$ after OVA injection in the ears on day 42. Mice in the negative control group were sham-sensitized with PBS. Mice in the other groups were sensitized with OVA, including the nasal challenges on days 35, 38 and 41. Mean \pm SEM ear swelling ( $n=9$ per group). a Statistically significant difference $(\mathrm{p}<0.01)$ as tested in a post hoc Dunnett's test after one-way ANOVA compared to the OVAsensitized PBS-treated group.

breve M-16V, L. plantarum NumRes8 or L. rhamnosus NumRes6.

Another characteristic of this animal model is the dominant presence of eosinophils in the BALF [33-35], a hallmark of allergic asthma in humans [36]. After treatment with $B$. breve $\mathrm{M}-16 \mathrm{~V}$, the bronchial influx of both eosinophils and lymphocytes in the BALF was reduced, while this was not observed after treatment with any of the other strains. Moreover, the levels of cytokines that are mainly produced by $\mathrm{T}$ cells were lower compared to the other treatments. This could indicate a reduced Th2 response, which would be in agreement with several papers that demonstrated modulations of Th2-allergic responses by certain probiotics [27, 32, 37]. Alternatively, it may be due to reduced numbers of cytokine-producing cells or a combination of both mechanisms. Oral treatment with $B$. breve M-16V also reduced serum OVA-specific IgE and OVA-IgG1, which also supports a reduced Th2 response. However, the reduction of Th2 cytokines in BALF and decreased serum levels of OVA-IgE and OVA-IgG1 were not only observed after oral treatment with $B$. breve M-16V: Th 2 cytokines IL- 4 and IL- 5 were also diminished after oral treatment with $B$. animalis NumRes252, while $B$. breve $\mathrm{M}-16 \mathrm{~V}, B$. animalis NumRes253 and L. rhamnosus NumRes6 reduced OVA-IgG1 levels, whereas all evaluated bacterial strains reduced 
OVA-IgE. It is known that Th2-produced IL-4 is essential for the switch to the IgG1 and IgE isotype. However, the results of the current study failed to show a $100 \%$ correlation between IL- 4 levels and OVA-IgG1 or OVA-IgE titers after treatment with each bacterial strain. After supplementation with $B$. breve $\mathrm{M}-16 \mathrm{~V}$ and $B$. animalis NumRes252, a correlation between reduced IL-4 in the BALF and reduced OVA-IgE and OVA-IgG1 was observed, whereas L. rhamnosus NumRes6 also decreased OVA-IgE and OVA-IgG1 without demonstrating a reduction in IL-4 in the BALF. Probably, other mechanisms are involved as well that affect these parameters. OVA-IgG2a, a Th1-related Ig titer, was not affected at all after supplementation with any of the bacterial strains.

In a subsequent experiment at another laboratory, the bacterial strains that reduced the response to methacholine and the number of eosinophils in BALF were evaluated for their effect on acute allergic skin reactions, another functional parameter of allergy. Of the 2 strains, only $B$. breve $\mathrm{M}-16 \mathrm{~V}$ reduced the acute allergic skin reaction, whereas $L$. plantarum NumRes8 was not effective.

This is the first report in which the reduction of these Th2 parameters appeared only partly predictive of either the reduction of bronchial eosinophils or lymphocytes (not affected by B. animalis NumRes253 or L. rhamnosus NumRes6), or reduction of airway response to methacholine (not affected by $B$. animalis NumRes253), or acute allergic skin reaction (only reduced by treatment with $B$. breve $\mathrm{M}-16 \mathrm{~V}$ ). This underlines the importance of including functional allergic parameters to aid in the selection of the best antiallergic bacterial strain. In addition, a reduced response to methacholine does not automatically imply an effect on bronchial cell influx as illustrated by the results obtained with L. rhamnosus NumRes6 (fig. 3, 4). Similarly, oral administration of LGG reduced airway inflammation, which included reduced numbers of eosinophils in BALF, while airway reactivity to methacholine in OVA-allergic BALB/c mice was not affected [26]. Mechanistic studies revealed that the induction of the airway response to methacholine and the development of eosinophil infiltration are differentially regulated [34, $35]$. Because the antiallergic effects of $B$. breve M-16V include both a reduced response to methacholine and eosinophil cell influx, it is highly likely that $B$. breve M-16V exerts its antiallergic effects via multiple mechanisms. $W$ ith regard to potential working mechanisms of $B$. breve $\mathrm{M}-16 \mathrm{~V}$, the finding that bifidobacteria possess immunostimulatory oligodeoxynucleotide DNA sequences (ISSODN) is of particular interest [38]. The ODN-BL07 sequence that was identified in Bifidobacterium longum

Probiotics Reduce Allergic Symptoms in Mice
BB536, is also present in B. breve M-16V [38]. Oral administration of ODN-BL07S, which was modified from ODN-BL07 by including a phosphorothioate backbone to provide nuclease resistance, was shown to reduce $\operatorname{IgE}$ production in vitro [38], to suppress $\mathrm{Th} 2$ responses when injected simultaneously with OVA in mice [39], and to favor the Th1/Th2 balance towards less Th2 and more Th1 in mice sensitized with OVA [40]. These immunomodulatory effects may be part of the mechanism underlying the antiallergic effects observed in the present study with B. breve M-16V, at least for the reduced Th2 parameters. A recent study demonstrated that total IgE, OVAIgE and OVA-IgG1 were reduced after oral administration of $B$. breve $\mathrm{M}-16 \mathrm{~V}$ in $\mathrm{BALB} / \mathrm{c}$ mice when supplemented for 21 days. In that study, the mice were sensitized with OVA twice on days 0 and 14 . Splenocytes were harvested on day 21 and cultured in the presence of OVA for another 7 days. The supernatants of the splenocytes from $B$. breve $\mathrm{M}-16 \mathrm{~V}$-supplemented mice showed a reduced amount of IL-4; no effects were observed for IL-10, IFN- $\gamma$ and transforming growth factor- $\beta$ (TGF- $\beta$ ) [41]. In agreement with that study were the reduced IL-4, reduced OVA-IgE and reduced OVA-IgG1 that were observed in the present study as well. In this study, IL-10 was decreased after treatment with $B$. breve $\mathrm{M}-16 \mathrm{~V}$, whereas it was not in the prevention study with $B$. breve M-16V [41]. Differences can be explained by the different setup and the reduced dose of $B$. breve $\mathrm{M}-16 \mathrm{~V}$ in the prevention study $\left(0.5 \times 10^{9} \mathrm{CFUs/day}\right)$, whereas in the present study $10^{9} \mathrm{CFUs} /$ day were administered.

There have been some studies with $B$. breve M-16V in infants that are relevant to the antiallergic effects observed in the present study. In a study with term infants (around 10 months) having AD, cow's milk hypersensitivity and less than $30 \%$ fecal bifidobacteria, they were treated with $B$. breve M-16V for 3 months; these infants were compared with a control group ( 9 months) that was not treated with $B$. breve M-16V. While in the B. breve M$16 \mathrm{~V}$ group total allergic scores decreased, allergic scores remained unaffected in the control group [42]. In a study with preterm infants $(n=11)$, treatment with $B$. breve M-16V enhanced serum levels of TGF- $\beta 1$ on days 14 and 28 after birth, whereas no effects were observed on serum IL-4, IL-5, IL-6, IFN- $\gamma$ or TNF- $\alpha$ compared to vehiclesupplemented infants $(\mathrm{n}=7)$. The enhanced serum TGF$\beta 1$ levels after $B$. breve M-16V administration were correlated with increased levels of the TGF- $\beta$ signaling molecule Smad3, while the levels of the antagonistic Smad7 remained unaffected [19]. These clinical data suggest that B. breve $\mathrm{M}-16 \mathrm{~V}$ may have some antiallergic effects al- 
though larger clinical trials are needed for definite confirmation. Moreover, the results in the above-mentioned clinical trials only partly reflect some of the antiallergic effects observed with $B$. breve $\mathrm{M}-16 \mathrm{~V}$ in the present study.

In this study, differential effects of specific species and strains of lactic acid bacteria were observed, as well as strain-specific effects of $2 B$. animalis strains. Molecular techniques confirmed that these $2 B$. animalis strains are indeed different (fig. 2). Thereby, the results of the present study contribute to the established concept that specific health effects of potential probiotics have to be demonstrated for each specific bacterial strain.

In conclusion, of the 4 bifidobacterium and 2 lactobacillus strains compared in the present study, $B$. breve $\mathrm{M}$ $16 \mathrm{~V}$ optimally reduced the response to methacholine and had the strongest attenuating effect on lung inflammation. The reduction of the allergic response with $B$. breve $\mathrm{M}-16 \mathrm{~V}$, reflected by the reduced response to methacholine and lung inflammation, coincided with reduced OVA-specific IgE and OVA-specific IgG1 levels. In a subsequent experiment at another laboratory, B. breve M$16 \mathrm{~V}$ reduced acute allergic skin reactions. Therefore, $B$. breve $\mathrm{M}-16 \mathrm{~V}$ was identified as the most promising probiotic candidate for further anti-allergic human nutritional intervention strategies.

\section{Acknowledgments}

The authors wish to thank T. Leusink-Muis and M. Haarman for their excellent technical support.

\section{References}

1 Beasley R: Worldwide variation in prevalence of symptoms of asthma, allergic rhinoconjunctivitis, and atopic eczema: ISAAC. Lancet 1998;351:1225-1232.

- 2 Wadonda-Kabondo N, Sterne JA, Golding J, Kennedy CT, Archer CB, Dunnill MG: A prospective study of the prevalence and incidence of atopic dermatitis in children aged 0-42 months. Br J Dermatol 2003;149:10231028.

-3 Strachan DP: Hay fever, hygiene, and household size. BMJ 1989;299:1259-1260.

$\checkmark 4$ Rook GA, Adams V, Hunt J, Palmer R, Martinelli R, Brunet LR: Mycobacteria and other environmental organisms as immunomodulators for immunoregulatory disorders. Springer Semin Immunopathol 2004;25: 237-255.

5 Romagnani S: The increased prevalence of allergy and the hygiene hypothesis: missing immune deviation, reduced immune suppression, or both? Immunology 2004;112: 352-363.

-6 Guarner F, Bourdet-Sicard R, Brandtzaeg P, Gill HS, McGuirk P, van Eden W, Versalovic J, Weinstock JV, Rook GA: Mechanisms of disease: the hygiene hypothesis revisited. Nat Clin Pract Gastroenterol Hepatol 2006; 3:275-284

-7 Bjorksten B, Naaber P, Sepp E, Mikelsaar M: The intestinal microflora in allergic Estonian and Swedish 2-year-old children. Clin Exp Allergy 1999;29:342-346.

-8 Bjorksten B, Sepp E, Julge K, Voor T, Mikelsaar M: Allergy development and the intestinal microflora during the first year of life. Allergy Clin Immunol 2001;108:516-520.
-9 Watanabe S, Narisawa Y, Arase S, Okamatsu H, Ikenaga T, Tajiri Y, Kumemura M: Differences in fecal microflora between patients with atopic dermatitis and healthy control subjects. J Allergy Clin Immunol 2003;111: 587-591.

10 Kalliomäki M, Kirjavainen P, Eerola E, Kero P, Salminen S, Isolauri E: Distinct patterns of neonatal gut microflora in infants in whom atopy was and was not developing. J Allergy Clin Immunol 2001;107:129-134.

11 Ouwehand A, Isolauri E, Salminen S: The role of the intestinal microflora for the development of the immune system in early childhood. Eur J Nutr 2002;41(suppl 1):I32-I37.

12 Kalliomäki M, Isolauri E: Role of intestinal flora in the development of allergy. Curr Opin Allergy Clin Immunol 2003;3:15-20.

13 Araya M, Gopal P, Lindgren SE, Lodi R, Oliver G, Saxelin ML, Servin AL: Health and Nutritional Properties of Probiotics in Food including Powder Milk with Live Lactic Acid Bacteria. Report of a joint FAO/WHO expert consultation on evaluation of health and nutritional properties of probiotics in food including powder milk with live lactic acid bacteria, Cordoba, 2001.

14 Isolauri E, Arvola T, Sutas Y, Moilanen E, Salminen S: Probiotics in the management of atopic eczema. Clin Exp Allergy 2000;30: 1604-1610.

15 Pessi T, Sutas Y, Hurme M, Isolauri E: Interleukin-10 generation in atopic children following oral Lactobacillus rhamnosus GG. Clin Exp Allergy 2000;30:1804-1808.

16 Rautava S, Kalliomäki M, Isolauri E: Probiotics during pregnancy and breast-feeding might confer immunomodulatory protection against atopic disease in the infant. J Allergy Clin Immunol 2002;109:119-121.
17 Pohjavuori E, Viljanen M, Korpela R, Kuitunen M, Tiittanen M, Vaarala O, Savilahti E: Lactobacillus GG effect in increasing IFN$\gamma$ production in infants with cow's milk allergy. J Allergy Clin Immunol 2004;114:131136.

18 Prescott SL, Dunstan JA, Hale J, Breckler L, Lehmann H, Weston S, Richmond P: Clinical effects of probiotics are associated with increased interferon- $\gamma$ responses in very young children with atopic dermatitis. Clin Exp Allergy 2005;35:1557-1564.

19 Fujii T, Ohtsuka Y, Lee T, Kudo T, Shoji H, Sato H, Nagata S, Shimizu T, Yamashiro Y: Bifidobacterium breve enhances transforming growth factor $\beta 1$ signaling by regulating Smad7 expression in preterm infants. J Pediatr Gastroenterol Nutr 2006;43:83-88.

20 Lee J, Seto D, Bielory L: Meta-analysis of clinical trials of probiotics for prevention and treatment of pediatric atopic dermatitis. J Allergy Clin Immunol 2008;121:116-121.

21 Kopp MV, Salfeld P: Probiotics and prevention of allergic disease. Curr Opin Clin Nutr Metab Care 2009;12:298-303.

-22 Kalliomäki M, Salminen S, Arvilommi H, Kero P, Koskinen P, Isolauri E: Probiotics in primary prevention of atopic disease: a randomised placebo-controlled trial. Lancet 2001;357:1076-1079.

23 Viljanen M, Savilahti E, Haahtela T, Juntunen-Backman K, Korpela R, Poussa T, Tuure T, Kuitunen M: Probiotics in the treatment of atopic eczema/dermatitis syndrome in infants: a double-blind placebo-controlled trial. Allergy 2005;60:494-500. 
-24 Grüber C, Wendt M, Sulser C, Lau S, Kulig K, Wahn U, Werfel T, Niggemann B: Randomized, placebo-controlled trial of Lactobacillus rhamnosus GG as treatment of atopic dermatitis in infancy. Allergy 2007;62: 1270-1276.

-25 Kopp MV, Hennemuth I, Heinzmann A, Urbanek R: Randomized, double-blind, placebo-controlled trial of probiotics for primary prevention: no clinical effects of Lactobacillus GG supplementation. Pediatrics 2008; 121:e850-e856.

-26 Blumer N, Sel S, Virna S, Patrascan CC, Zimmermann S, Herz U, Renz H, Garn H: Perinatal maternal application of Lactobacillus rhamnosus GG suppresses allergic airway inflammation in mouse offspring. Clin Exp Allergy 2007;37:348-357.

27 Feleszko W, Jaworska J, Rha RD, Steinhausen S, Avagyan A, Jaudszus A, Ahrens B, Groneberg DA, Wahn U, Hamelmann E: Probioticinduced suppression of allergic sensitization and airway inflammation is associated with an increase of $\mathrm{T}$ regulatory-dependent mechanisms in a murine model of asthma. Clin Exp Allergy 2007;37:498-505.

28 Lane D: 16S/23S rRNA sequencing; in Stackebrandt E, Goodfellow M (eds): Nucleic Acid Techniques in Bacterial Systematics. New York, Wiley, 1991, pp 115-175.

-29 Tilsala-Timisjärvi A, Alatossava T: Development of oligonucleotide primers from the 16S-23S rRNA intergenic sequences for identifying different dairy and probiotic lactic acid bacteria by PCR. Int J Food Microbiol 1997;35:49-56.
30 Hamelmann E, Schwarze J, Takeda K, Oshiba A, Larsen GL, Irvin CG, Gelfand EW: Noninvasive measurement of airway responsiveness in allergic mice using barometric plethysmography. Am J Respir Crit Care Med 1997;156:766-775.

31 Vos AP, van Esch BC, Stahl B, M'Rabet L, Folkerts G, Nijkamp FP, Garssen J: Dietary supplementation with specific oligosaccharide mixtures decreases parameters of allergic asthma in mice. Int Immunopharmacol 2007;7:1582-1587.

32 Forsythe P, Inman MD, Bienenstock J: Oral treatment with live Lactobacillus reuteri inhibits the allergic airway response in mice. Am J Respir Crit Care Med 2007;175:561569.

33 De Bie JJ, Hessel EM, Van Ark I, Van Esch B, Hofman G, Nijkamp FP, Van Oosterhout AJ: Effect of dexamethasone and endogenous corticosterone on airway hyperresponsiveness and eosinophilia in the mouse. $\mathrm{Br}$ Pharmacol 1996;119:1484-1490.

34 Hessel EM, Van Oosterhout AJ, Van Ark I, Van Esch B, Hofman G, Van Loveren H, Savelkoul HF, Nijkamp FP: Development of airway hyperresponsiveness is dependent on interferon- $\gamma$ and independent of eosinophil infiltration. Am J Respir Cell Mol Biol 1997; 16:325-334.

35 Foster PS, Hogan SP, Ramsay AJ, Matthaei KI, Young IG: Interleukin 5 deficiency abolishes eosinophilia, airways hyperreactivity, and lung damage in a mouse asthma model. J Exp Med 1996;183:195-201.

36 Wardlaw AJ, Brightling C, Green R, Woltmann G, Pavord I: Eosinophils in asthma and other allergic diseases. Br Med Bull 2000;56:985-1003.
37 Fujiwara D, Inoue S, Wakabayashi H, Fujii T: The anti-allergic effects of lactic acid bacteria are strain dependent and mediated by effects on both Th1/Th2 cytokine expression and balance. Int Arch Allergy Immunol 2004;135:205-215.

38 Takahashi N, Kitazawa H, Shimosato T, Iwabuchi N, Xiao JZ, Iwatsuki K, Kokubo S, Saito T: An immunostimulatory DNA sequence from a probiotic strain of Bifidobacterium longum inhibits IgE production in vitro. FEMS Immunol Med Microbiol 2006; 46:461-469.

39 Takahashi N, Kitazawa H, Iwabuchi N, Xiao JZ, Miyaji K, Iwatsuki K, Saito T: Immunostimulatory oligodeoxynucleotide from Bifidobacterium longum suppresses Th2 immune responses in a murine model. Clin Exp Immunol 2006;145:130-138.

40 Takahashi N, Kitazawa H, Iwabuchi N, Xiao JZ, Miyaji K, Iwatsuki K, Saito T: Oral administration of an immunostimulatory DNA sequence from Bifidobacterium longum improves Th1/Th2 balance in a murine model. Biosci Biotechnol Biochem 2006;70: 2013-2017.

41 Inoue Y, Iwabuchi N, Xiao JZ, Yaeshima T, Iwatsuki K: Suppressive effects of Bifidobacterium breve strain M-16V on T-helper type 2 immune responses in a murine model. Biol Pharm Bull 2009;32:760-763.

42 Taniuchi S, Hattori K, Yamamoto A, Sasai M, Hatano Y, Kojima T, Kobayashi Y, Iwamoto H, Yaeshima T: Administration of bifidobacterium to infants with atopic dermatitis: changes in fecal microflora and clinical symptoms. J Appl Res 2005;5:387-396. 